\title{
Profiling of Metabolites from Human Intervertebral Disc through Gas Chromatography - Mass Spectrometry
}

\author{
R. Veera Ranjani ${ }^{1}$, N. Senthil ${ }^{*}$, M. Raveendran ${ }^{1}$, S. Vellaikumar', \\ R. Gnanam ', M. K. Rishi' ${ }^{2}$ and S. Rajasekaran ${ }^{2}$ \\ 'Centre for Plant Molecular Biology and Biotechnology, Tamil Nadu Agricultutal University, \\ Coimbatore-641003,Tamil Nadu, India; rajaranji@gmail.com, senthil_natesan@tnau.ac.in, \\ raveendrantnau@gmail.com, vellai1973@gmail.com, rgnanam2000@yahoo.com \\ 2Department of Orthopaedics and Spine Surgery, Ganga Hospital, Coimbatore, Tamil Nadu, \\ India; rishiortho@gmail.com, rajasekaran.orth@gmail.com
}

\begin{abstract}
This work aims to identify the metabolites present in the human Intervertebral Disc (IVD). Metabolomic analysis of human IVD tissue has not been extensively done to date. Knowledge on the metabolites present in the IVD tissue in humans is very limited and many compounds are yet to be identified. In this study, we have carried out the metabolic profiling for human IVD through Gas Chromatography/Mass Spectrometry (GC/MS). This is the first initial study that has compared the metabolites of control and diseased IVD. We have identified 75 different chemical compounds in IVD, and also metabolites that are unique to the diseased IVD, suggesting that some of these metabolites might play a role in disc degenerative disease.
\end{abstract}

Keywords: DDD, Human, Intervertebral Disc, Metabolite Profiling

\section{Introduction}

Low Back Pain (LBP) is a global health problem in which more than $40 \%$ is caused by lumbar intervertebra ${ }^{1}$ disc degeneration 1 and one of the most important health care issues today. About $60 \%$ and $80 \%$ of the global population experiences LBP at least once in their lifetime ${ }^{2}$. The annual prevalence ranges from $15 \%$ to $45 \%$ with the point prevalence averaging $30 \%{ }^{3}$. Only a small proportion (approximately 20\%) of LBP cases can be attributed with reasonable certainty to a pathologic or anatomical entity. Thus, diagnosing the cause of LBP represents the biggest challenge for doctors in this field. Recent reports are showing enough evidences to the involvement of genetic factors and a number of genes like Vitamin D Receptor
$(\mathrm{VDR})^{4}$, Collagen - factors $^{5}$, Interleukins ${ }^{6}$, MMP3 (Matrix metalloproteinase-3) ${ }^{7}$, aggrecan ${ }^{8}$ and cartilage intermediate layer protein ${ }^{2}$ are reported to be associated with DDD. A few candidate genes, which have a weak association with DDD (viz., (Vitamin D receptor) VDR, (collagen IX A2) COL9A2, (collagen IX A3) COL9A3, (Matrix metalloprotease-3) MMP3 and Aggrecan), have been identified using genetic polymorphisms ${ }^{9}$. However, functional studies of candidate genes will be an important step for testing whether a candidate gene is truly associated with Disc Degenerative Disease (DDD) or not.Hence systematic analysis of genes expressed in IVD through high throughput genomic tools viz., microarrays, proteomics and metabolomics are needed which will shed light on the major events associated with DDD and lead

${ }^{*}$ Author for correspondence 
to the identification of metabolic pathways involved in this degeneration.

\section{Materials and Methods}

The IVD of control and patient were obtained from spine surgery unit and used after getting permission from Ethical Clearance Committee with informed consent. For method development and validation, a representative control and affected disc tissue was snap-frozen immediately following surgery and then stored at $-80^{\circ} \mathrm{C}$. About $20 \mathrm{mg}$ of the stored tissue weighed accurately and ground with liquid nitrogen. Then $1 \mathrm{~mL}$ of a mono-phasic mixture of chloroform/methanol/water in ratio of 20:50:20 (v/v/v) was added to each sample ${ }^{10}$. The samples were ultrasonicated in an ultra-sonicator bath at ambient temperature $\left(24-28^{\circ} \mathrm{C}\right)$ for $100 \mathrm{~min}$ and then vortex-mixed for $2 \mathrm{~min}$. The samples were subsequently centrifuged at $12000 \mathrm{rpm}$ for $10 \mathrm{~min}$ and the supernatant was collected separately from each sample in different tubes. The collected supernatant was concentrated to complete dryness at $50^{\circ} \mathrm{C}$ for $30 \mathrm{~min}$. A $100 \mu \mathrm{L}$ of toluene (kept anhydrous with sodium sulfate) was added to each of the sample extracts, vortex-mixed for $5 \mathrm{~min}$ and again evaporated to complete dryness using vacuum evaporator in order to eliminate any trace amount of water which might interfere with the GC/MS analysis.

\subsection{Derivatization Procedure}

The dried samples were then derivatized by adding $100 \mu \mathrm{L}$ of MSTFA with $1 \%$ TMCS to each sample. The samples were then vortex-mixed for $2 \mathrm{~min}$ and incubated at $70^{\circ} \mathrm{C}$ for $30 \mathrm{~min}$. After incubation, samples were again vortexmixed for 2 min and then transferred to glass vials for GC/MS analysis.

\subsection{GC/MS Analysis}

The analysis was performed on a Thermo GC - Trace Ultra Ver: 5.0, Thermo Ms DSQ II. A DB 5 - MS capillary standard non - polar column ( $30 \mathrm{x} 0.25 \mathrm{~mm} ; 0.25 \mu \mathrm{m}$ flim thickness, Thermo Scientific). Helium was used as the carrier gas at $1.0 \mathrm{~mL}$ per min and the injector split ratio was set to 1:5. An injection volume of $1 \mu \mathrm{l}$ was used and the solvent cut off time was $5 \mathrm{~min}$.

The injector and source temperatures were kept at $250^{\circ} \mathrm{C}$ and $200^{\circ} \mathrm{C}$, respectively. The oven temperature was kept at $60^{\circ} \mathrm{C}$ for $3 \mathrm{~min}$, increased at $7^{\circ} \mathrm{C} \mathrm{min}^{-1}$ to $140^{\circ} \mathrm{C}$ where it was held for $4 \mathrm{~min}$ and further increased at $5^{\circ} \mathrm{C}$ $\min ^{-1}$ to $310^{\circ} \mathrm{C}$ where it remained for $6 \mathrm{~min}$. The mass spectrometer was operated in Electron Impact (EI) ionization mode at $70 \mathrm{eV}$. Data acquisition was performed in the full scan mode from $\mathrm{m} / \mathrm{z} 50$ to 650 with a scan time of $0.5 \mathrm{~s}$.

\section{Results}

\subsection{Metabolite Identification and Data Processing}

GC/MS analysis from this study led to the identification of 75 different metabolites (Figure 1 and 2) belonging

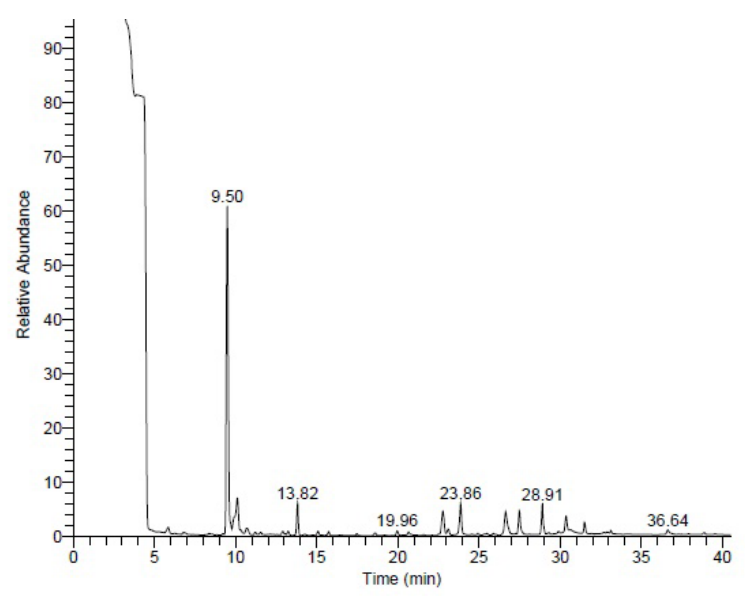

Figure 1. GC/MS chromatograms of control intervertebral disc.

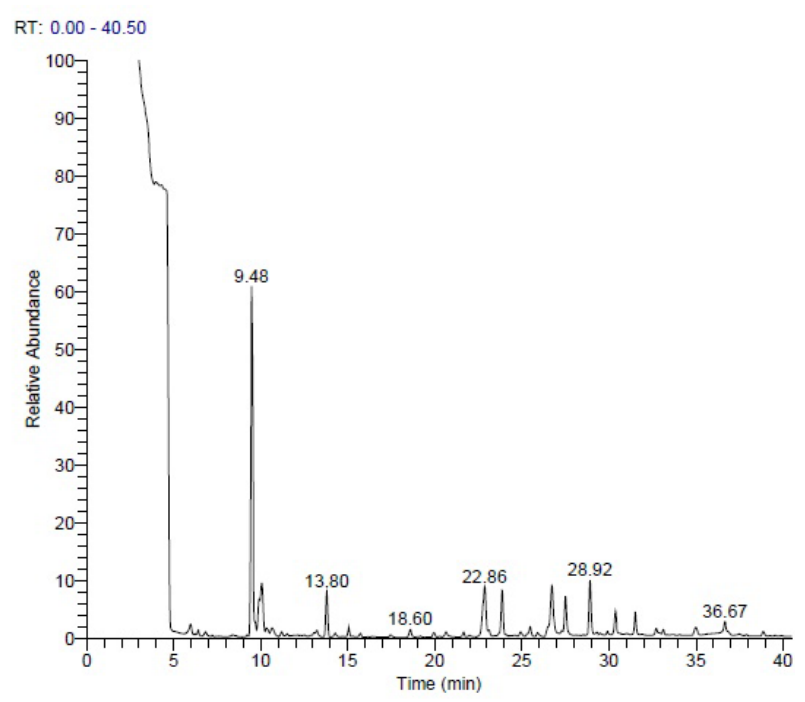

Figure 2. GC/MS chromatograms of degenerated intervertebral disc. 
to diverse chemical classes such as amino acids, organic acids, fatty acids, phenolics, silanes, nitriles, ethers, amines, azides, hydrocarbons, alcohols, carbonyl compounds, heterocyclic compounds, spiro compounds and metal complex (Table 1 and 2). The identities of selected metabolites were recognized by the NIST mass spectral library.

\section{Discussion}

GC/MS-based metabolomics is a powerful tool to elucidate the impact of low back pain thereby providing novel insights into the pathomechanism underlying disc degenerative disease. This GC/MS based metabolomic approaches cover a large number of novel compounds.

Table 1. Identified metabolites from control intervertebral disc tissue through GC/MS against NIST library search

\begin{tabular}{|c|c|c|c|}
\hline S. No & Metabolites & $\begin{array}{l}\text { Retention } \\
\text { time }\end{array}$ & Chemical groups identified \\
\hline 1 & Cyclopropanecarboxylic acid, pent-2-en-4-ynyl ester & 3.06 & Organic acid and its derivatides \\
\hline 2 & N1-(Formyl)-N2-(1-oxobut-2-en-1-yl)hydrazide & 3.45 & Azides \\
\hline 3 & 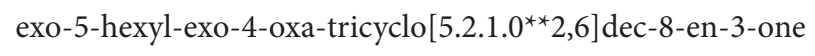 & 4.43 & Carbonyl compounds \\
\hline 4 & Methoxy-phenyl oxime & 4.92 & Carbonyl compounds \\
\hline 5 & D-Lactic acid-DITMS & 5.28 & organic acid and its derivatives \\
\hline 6 & $\begin{array}{l}\text { Methyl 3-((aminocarbonyl)amino)-2-cyano-3- } \\
\text { phenylpropenoate }\end{array}$ & 5.87 & Organic acid and its derivatides \\
\hline 7 & 2-[5-(2-hydroxyethyl)-2-thienyl]-4,4-dimethyloxazoline & 6.28 & Heterocyclic compounds \\
\hline 8 & 2-methyl Decane & 6.77 & Hydrocarbons \\
\hline 9 & $\begin{array}{l}\text { 1,2-Dioxetane, 3,4,4-trimethyl-3-[((trimethylsilyl)oxy] } \\
\text { methyl]1,2-Dioxetane }\end{array}$ & 7.38 & Silanes \\
\hline 10 & 5,8-Diethoxy-7-methoxyquinoline & 8.05 & Heterocyclic compounds \\
\hline 11 & Octanoic acid, trimethylsilyl ester & 8.4 & fatty acid (S) \\
\hline 12 & 1-Tert-buthoxy-6-trimethylsilyloxyhexane & 8.79 & Ethers \\
\hline 13 & Bis(trimethylsilyl) 3-Ketovaleric acid & 9.22 & Amino acids \\
\hline 14 & $\begin{array}{l}\text { Tris(hydroxymethyl)aminomethane,O,O'O"'- } \\
\text { tris(trimethylsilyl) ether }\end{array}$ & 9.5 & Ethers \\
\hline 15 & 3-hydroxy Benzocycloheptene & 10.12 & Hydrocarbons \\
\hline 16 & 4-Methyl-6-cyanothieno[2,3-b]pyridine & 10.31 & Heterocyclic compounds \\
\hline 17 & [(2-deuterio)-s-isobutyl]-2-propenyl-sulfoxide & 10.73 & Hydrocarbon \\
\hline 18 & N-Acryloylmorpholine & 11.21 & Heterocyclic compounds \\
\hline 19 & 4,5-Dihydro-4,5-trans-di-n-propyl-2-ethoxyimidazole & 11.54 & Heterocyclic compounds \\
\hline 20 & Docosane & 12.16 & Hydrocarbon \\
\hline 21 & N-Nitrosomethylethylamine (à-D2) & 12.57 & Amines \\
\hline 22 & [6-(4-tert-Butylphenyl)-1,3,5-hexatriynyl]trimethylsilane & 12.9 & Silanes \\
\hline 23 & Hexadecane (CAS) & 13.24 & Hydrocarbon \\
\hline 24 & 3-phenyl-1,2-naphthoquinone & 13.82 & Hydrocarbon \\
\hline 25 & Dodecanoic acid (CAS) & 14.26 & fatty acid (S) \\
\hline 26 & Eicosane, 10-methyl- (CAS) & 14.72 & Hydrocarbon \\
\hline 27 & Isopropyl Dodecanoate & 15.09 & Organic acid and its derivatives \\
\hline 28 & 2-Methoxy-8-Chloro-Dibenzofuran & 15.27 & Heterocyclic compounds \\
\hline 29 & Eicosane, 2-methyl- (CAS) & 15.74 & Hydrocarbon \\
\hline
\end{tabular}

Continued... 


\begin{tabular}{|c|c|c|c|}
\hline 30 & Oxirane, hexyl- (CAS) & 15.98 & Cyclic ether (Ethylene oxide) \\
\hline 31 & Malonic acid, dodecyl 2-ethylbutyl ester & 16.19 & Organic acid and its derivatives \\
\hline 32 & 1-methoxymethyl-4-methylnaphthalene & 16.41 & Hydrocarbon \\
\hline 33 & Nonadecanoic acid, 18-oxo-, methyl ester (CAS) & 17.25 & fatty acid $(S)$ \\
\hline 34 & 17-Methyl-9-oxo-10-nor-14à-4,5-nitrilomorphinan & 17.46 & Heterocyclic compounds \\
\hline 35 & Tetradecanoic acid (CAS) & 18.59 & fatty acid (S)(Myristic acid \\
\hline 36 & 2-(o-Hydroxymethylbenzyl)naphtho[2,3-b] thiophene & 19.19 & Heterocyclic compounds \\
\hline 37 & Heneicosane & 19.96 & Hydrocarbon \\
\hline 38 & (15à)-phylloclad-16-ene-15-carbaldehyde & 20.65 & Carbonyl compounds \\
\hline 39 & Eicosane & 21.55 & Hydrocarbon \\
\hline 40 & 1-Hydroxy-17-(1-oxoethyl)-2-oxa-androst-4-en-3-one & 22.14 & Carbonyl compounds \\
\hline 41 & $\begin{array}{l}\text { 1,2-Benzenedicarboxylic acid, bis(2-methylpropyl) ester } \\
\text { (CAS) }\end{array}$ & 22.79 & Organic acid and its derivatives \\
\hline 42 & 2-Methoxycarbonyl-3-phenylsulfonylhydroquinone & 23.1 & Hydrocarbon \\
\hline 43 & 7,9-Di-tert-butyl-1-oxaspiro(4,5)deca-6,9-diene-2,8-dione & 23.86 & Carbonyl compounds \\
\hline 44 & Bis $[1,2$-di(2-thienyl)-1,2-ethenedithiolene]nickel & 24.57 & Metal complex \\
\hline 45 & Dibutyl phthalate & 24.93 & organic acid and its derivatives \\
\hline 46 & 4-(4-Fluorophenyl)-2-methyl-6-methylthiobenzonitrile & 25.51 & Hydrocarbon \\
\hline 47 & Diethyl [2- ( 4'-methylphenyl) ethyl] phosphonate & 25.89 & Organic acid and its derivatives \\
\hline 48 & 2,2-Dichlorocyclobuta[a]cyclopent [3,4-a]azulenone & 26.64 & Carbonyl compounds \\
\hline 49 & 1,3-Dimethoxy-5,7-dihydrodibenz[c,e]oxepine & 27.48 & Heterocyclic compounds \\
\hline 50 & Hentriacontane & 28.12 & Hydrocarbon \\
\hline 51 & Octadecanoic acid, propyl ester (CAS) & 28.31 & Fatty acid (S) (Stearic acid \\
\hline 52 & 10,10-dimethylanthrone hydrazone & 28.53 & Carbonyl compounds \\
\hline 53 & 1,3-Dimethoxy-5,7-dihydrodibenz[c,e]oxepine & 28.91 & Heterocyclic compounds \\
\hline 54 & 9,10-Dihydrocyclobuta[a]triphenylene-11,12-dione & 29 & Carbonyl compounds \\
\hline 55 & Pulchelstyrene D & 29.91 & Hydrocarbon \\
\hline 56 & 3,7-dimethoxy-1,9-dimethyldibenzofuran-4-carbaldehyde & 30.36 & Carbonyl compounds \\
\hline 57 & $\begin{array}{l}\text { 3,18-Epoxyandrosta-5,7-dien-17-ol, 4,4-dimethyl-3- } \\
\text { methoxy- (13á) }\end{array}$ & 31.25 & Epoxide (Hydrocarbons) \\
\hline 58 & 3,7-dimethoxy-1,9-dimethyldibenzofuran-4-carbaldehyde & 31.5 & Carbonyl compounds \\
\hline 59 & $\begin{array}{l}\text { 2áBenzyl-8-oxo-4,6-dimethyl-3,5,7-trioxatetracyclo[7.2.1. } \\
0(4,11) .0(6,10)] \text { dodecane }\end{array}$ & 31.94 & Hydrocarbon \\
\hline 60 & Pentacosane & 32.87 & Hydrocarbon \\
\hline 61 & Di-(2-ethylhexyl)phthalate & 33.14 & Organic acid and its derivatives \\
\hline 62 & Hexadecane, 2,6,10,14-tetramethyl- (CAS) & 33.32 & Hydrocarbon \\
\hline 63 & Nonacosane (CAS) & 33.59 & Hydrocarbon \\
\hline 64 & 03027205002 Flavone & 34.27 & Carbonyl compounds \\
\hline 65 & 10-Benzyloxy-1,8-dihydroxy-9(10H)-anthracenone & 35.21 & Carbonyl compounds \\
\hline 66 & 2-Pentoxy-tetrahydropyran & 35.59 & Heterocyclic compounds \\
\hline 67 & N-(5à-Cholestan-3á-yl)-acetamide & 36.17 & Organic acids and its derivatives \\
\hline
\end{tabular}

Continued ... 


\begin{tabular}{|c|c|c|c|}
\hline 68 & $\begin{array}{l}\text { Octadecanoic acid, 2-hydroxy-1-(hydroxymethyl)ethyl ester } \\
\text { (CAS) }\end{array}$ & 36.64 & Fatty acid \\
\hline 69 & Tetratetracontane (CAS) & 37.31 & Hydrocarbon \\
\hline 70 & $\begin{array}{l}\text { 1,4-Cyclohexadiene-1,2-dicarboxylic acid, 4,5-dimethyl-, } \\
\text { dimethyl ester }\end{array}$ & 37.92 & Organic acids and its derivatives \\
\hline 71 & $\begin{array}{l}\text { Acetic acid, } 4,5 \text {-dihydroxy-10,13-dimethyl-3- } \\
\text { oxohexadecahydrocyclop enta[a]phenanthren-17-yl ester }\end{array}$ & 38.21 & Organic acids and its derivatives \\
\hline 72 & Methyl 4-(4-methoxybenzoyl)-4-methyl-pent-2-enoate & 38.5 & Organic acids and its derivatives \\
\hline 73 & 13-Docosenamide, $(Z)$ - & 38.87 & Organic acids and its derivatives \\
\hline 74 & $\begin{array}{l}\text { 2-[3-(Aminomethyl)-5,7-Dimethyl-1-Adamantyl] } \\
\text { Ethanamine }\end{array}$ & 39.56 & Amines \\
\hline 75 & Dihydromonticamine & 39.93 & Amines \\
\hline
\end{tabular}

Table 2. Identified metabolites from degenerated intervertebral disc tissue through GC/MS against NIST library search

\begin{tabular}{|c|c|c|c|}
\hline S. No & Metabolites & $\begin{array}{l}\text { Retention } \\
\text { time }\end{array}$ & Chemical groups identified \\
\hline 1 & 1-Benzyloxymethyl-1-Hydroxymethyl-2,5- Cyclohexadiene & 3.1 & Hydrocarbon \\
\hline 2 & 4-Chloro-4-(phenylsulfinyl)-3-heptanol isomer & 3.51 & Alcohols \\
\hline 3 & $\begin{array}{l}\text { (6á)-8a-(3',3'-Dimethylbut-1'-ynyl)-3,4,4a,5,6,8a-hexahydro-6- } \\
\text { methoxy-3,3,6-trimethylnaphthalen-1(2H)-one }\end{array}$ & 4.65 & Carbonyl compound \\
\hline 4 & 4-[3-(Trimethylsilyl)-2-propinyl]oxy-2-butynoic acid & 5.09 & Organic acid and its derivatives \\
\hline 5 & D-Lactic acid-DITMS & 6 & Organic acid and its derivatives \\
\hline 6 & 2-[5-(2-hydroxyethyl)-2-thienyl]-4,4-dimethyloxazoline & 6.41 & Heterocyclic compounds \\
\hline 7 & Ethyl 3-(Trimethylsilyl)Propanoate \# & 6.84 & Organic acid and its derivatives \\
\hline 8 & $\begin{array}{l}\text { 3,7-Dioxa-2,8-disilanonane, 2,2,8,8-tetramethyl-5- } \\
\text { [(trimethylsilyl)oxy]- (CAS) }\end{array}$ & 7.22 & Silanes \\
\hline 9 & 2-[4(or 5)-(2-Phenylimidazolyl)]propionitrile & 7.59 & Nitriles \\
\hline 10 & 3-Phenylnon-4-en-3-ol & 8.06 & Alcohols \\
\hline 11 & Octanoic acid, trimethylsilyl ester & 8.43 & Organic acid and its derivatives \\
\hline 12 & $\begin{array}{l}\text { 9,12,15-Octadecatrienoic acid,2-[(trimethylsilyl)oxy]-1- } \\
\text { [[(trimethylsilyl)oxy]methyl]ethyl ester, (Z,Z,Z)- (CAS) }\end{array}$ & 8.81 & Organic acid and its derivatives \\
\hline 13 & 2-Aminoethanol, $\mathrm{N}$-acetyl-, trimethylsilyl ether & 9.04 & Ethers \\
\hline 14 & 3-Oxovaleric acid TMS ether TMS ester & 9.22 & Amino acids \\
\hline 15 & 5-Benzoyl-1,2,3,4-tetrahydronaphthalene & 9.48 & Hydrocarbon \\
\hline 16 & Dibenzo[c,e]thiin-2-thione & 10.05 & Carbonyl compound \\
\hline 17 & 4-Methyl-6-cyanothieno[2,3-b]pyridine & 10.31 & Heterocyclic compound \\
\hline 18 & Eicosane (CAS) & 10.65 & Hydrocarbon \\
\hline 19 & N-Acryloylmorpholine & 11.21 & Heterocyclic compound \\
\hline 20 & Hexadecane, 2,6,11,15-tetramethyl- (CAS) & 11.52 & Hydrocarbon \\
\hline 21 & 1,2-Difluoro-3,4,5-trimethylbenzene & 12.12 & Hydrocarbon \\
\hline 22 & N,N'-Ditrityl-1,5-diaminopentane & 12.51 & Hydrocarbon \\
\hline
\end{tabular}

Continued ... 


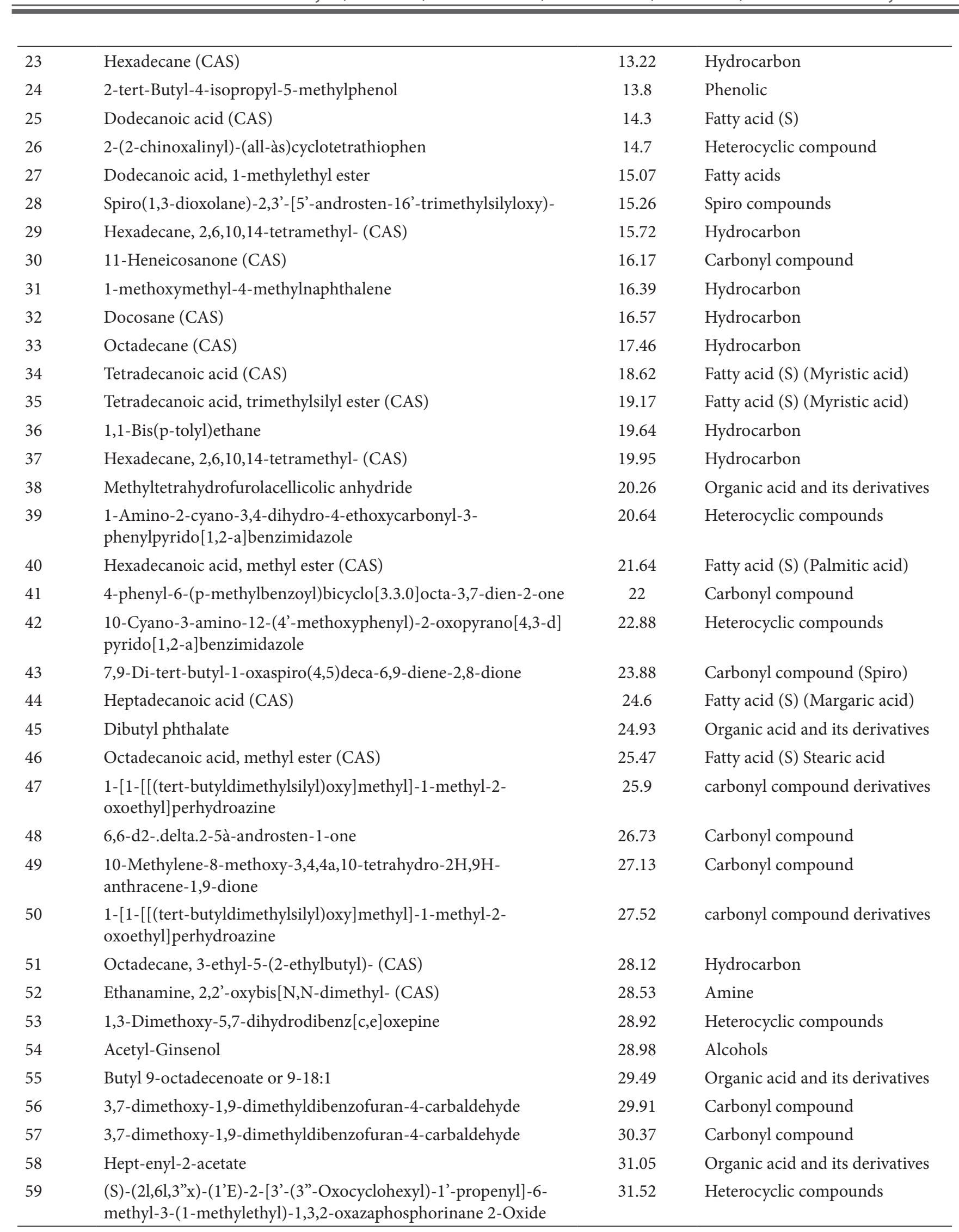

Continued ... 


\begin{tabular}{|c|c|c|c|}
\hline 60 & $\begin{array}{l}\text { 2áBenzyl-8-oxo-4,6-dimethyl-3,5,7-trioxatetracyc } \\
\text { lo[7.2.1.0(4,11).0(6,10)]dodecane }\end{array}$ & 31.93 & Hydrocarbon \\
\hline 61 & Hexadecanoic acid, 2-hydroxy-1-(hydroxymethyl)ethylester & 32.7 & Organic acid and its derivatives \\
\hline 62 & Di-(2-ethylhexyl)phthalate & 33.13 & Organic acid and its derivatives \\
\hline 63 & Docosane, 11-decyl- (CAS) & 33.34 & Hydrocarbon \\
\hline 64 & erythro-1,2-Epoxy-2-methyl-3-heptanol & 33.68 & Alcohols \\
\hline 65 & $\begin{array}{l}\text { 7-(4'-Nitrophenyl)-5-imino-2-methyl-5H-thiazolo[3,2-a] } \\
\text { pyridine-6-carbonitrile }\end{array}$ & 34.05 & Heterocyclic compounds \\
\hline 66 & $\begin{array}{l}\text { 3-Formyl-1-oxyl-4-(pyren-1'-yl)-2,2,5,5-tetramethyl-2,5- } \\
\text { dihydro-1H-pyrrole }\end{array}$ & 34.52 & Heterocyclic compounds \\
\hline 67 & 1'-Benzyl-3-methoxynaphtho[16,17-b]estra-1,3,5(6)-triene & 34.99 & Hydrocarbon \\
\hline 68 & Cholan-24-oic acid, 3,12-dihydroxy-, (3à,5á,12à)- (CAS) & 35.66 & Organic acids \\
\hline 69 & Octadecanoic acid, 2-hydroxy-1-(hydroxymethyl)ethylester & 36.67 & Organic acid and its derivatives \\
\hline 70 & 3,5-dicyano-2,6-di(m-tolyl)-1-methylpyridine-4(1H)-thione & 37.5 & Heterocyclic compounds \\
\hline 71 & 2,6-Diisopropylanisole & 37.91 & Phenolic \\
\hline 72 & 13-Docosenamide, $(Z)$ - & 38.87 & Organic acid and its derivatives \\
\hline 73 & 2,2,5,5-Tetramethyl-4-(p-methoxyphenyl)-3-oxazoline & 38.97 & Heterocyclic compounds \\
\hline 74 & Benzoic acid, 2,4-dimethyl-, (3,5-dimethylphenyl)methylester & 39.56 & Organic acid and its derivatives \\
\hline 75 & Hexadecanoic acid, hexadecyl ester (CAS) & 39.93 & Organic acid and its derivatives \\
\hline
\end{tabular}

Unlike to proteomics, metabolomics demands a large amount of manual evaluation like the validation and peak correction of identified metabolites. Mass spectrometric analysis could identify the metabolites present in the disc tissue sample through NIST library search.

Interestingly, the presence of lactic acid in our study indicates that lactic acid might play a role in disc degeneration. Intervertebral disc is the largest avascular tissue in the body, its metabolism is mainly anaerobic, and thus lactate is produced at a significant rate. The higher lactate concentration reduces the $\mathrm{pH}$ in the disc ${ }^{11,12}$. Experimental data from other studies have showed that acidic $\mathrm{pH}$ adversely affect the supply of nutrients to IVD, cellular activity of IVD ${ }^{13,14}$, synthesis of proteoglycans (which play a major role in the load bearing capacity of the disc) $)^{12}$, integrity of extracellular matrix of the IVD, even IVD viability ${ }^{15}$, and thus acidification of IVD due to lactic acid may play a role in disc degeneration ${ }^{13,14}$.

The presence of lactic acid in both control and degenerated disc indicates that quantification of lactic acid is necessary for correlating the quantity of lactic acid with the causation of IVD degeneration. Similarly further confirmatory studies are required to ascertain whether the compounds found in our study play real roles in disc degenerative disease. Our future studies will concentrate on the quantification of the metabolites from both control and affected tissue for obtaining valuable insights about the IVD degeneration.

\section{Conclusion}

Our GC/MS-based metabolic profiling study has profiled the metabolites present in both control and degenerated human IVD. Our study revealed that some of these metabolites present in the human IVD might play an important role in disc degenerative diseases.

\section{References}

1. Wu N, Chen J, Liu H, Zhao L, Liu S, Liu J, Su X, Wu W, Cong J, Qiu G, Wu Z.. The involvement of ADAMTS-5 genetic polymorphisms in predisposition and diffusion tensor imaging alterations of lumbar disc degeneration. J Orthop Res. 2014.

2. Seki S, Kawaguchi Y, Chiba K, Mikami Y, Kizawa H, Oya T, et al. A functional SNP in CILP, encoding cartilage intermediate layer protein, is associated with susceptibility to lumbar disc disease. Nat Genet. 2005 Jun; 37(6):607-12.

3. Andersson GB. Epidemiological features of chronic lowback pain. The Lancet. 1999; 354(9178):581-5. 
4. Videman T, Leppävuori J, Kaprio J, Battie MC, Gibbons LE, Peltonen L, et al. Intragenic polymorphisms of the vitamin $\mathrm{D}$ receptor gene associated with intervertebral disc degeneration. Spine. 1998; 23(23):2477-85.

5. Feng $\mathrm{H}$, Danfelter $\mathrm{M}$, StrÃ ķmqvist B, Heineg ̃̃̃̃rd D. Extracellular matrix in disc degeneration. J Bone Joint Surg. 2006; 88(suppl_2):25-9.

6. Solovieva S, Lohiniva J, Leino-Arjas P, Raininko R, Luoma $\mathrm{K}$, Ala-Kokko L, et al. Intervertebral disc degeneration in relation to the COL9A3 and the IL-1ss gene polymorphisms. Eur Spine J. 2006; 15(5):613-9.

7. Goupille P, Jayson MI, Valat J-P, Freemont AJ. Matrix metalloproteinases: the clue to intervertebral disc degeneration? Spine. 1998; 23(14):1612-26.

8. Kawaguchi Y, Osada R, Kanamori M, Ishihara H, Ohmori $\mathrm{K}$, Matsui $\mathrm{H}$, et al. Association between an aggrecan gene polymorphism and lumbar disc degeneration. Spine. 1999; 24(23):2456-60.

9. Chan D, Song Y, Sham P, Cheung KM. Genetics of disc degeneration. Eur Spine J. 2006 Aug;15 Suppl 3:S317-25.
10. Mal M, Koh PK, Cheah PY, Chan ECY. Development and validation of a gas chromatography/mass spectrometry method for the metabolic profiling of human colon tissue. Rapid Comm Mass Spectrom. 2009; 23(4):487-94.

11. Diamant B, Karlsson J, Nachemson A. Correlation between lactate levels and $\mathrm{pH}$ in discs of patients with lumbar rhizopathies. Experientia. 1968; 24(12):1195-6.

12. Ohshima $\mathrm{H}$, Urban J. The effect of lactate and $\mathrm{pH}$ on proteoglycan and protein synthesis rates in the intervertebral disc. Spine. 1992; 17(9):1079-82.

13. Ishihara H, Urban JP. Effects of low oxygen concentrations and metabolic inhibitors on proteoglycan and protein synthesis rates in the intervertebral disc. J Orthop Res. 1999; 17(6):829-35.

14. Razaq S, Wilkins RJ, Urban JP. The effect of extracellular $\mathrm{pH}$ on matrix turnover by cells of the bovine nucleus pulposus. Eur Spine J. 2003;12(4):341-9.

15. Bibby S, Urban J. Effect of nutrient deprivation on the viability of intervertebral disc cells. Eur Spine J. 2004; 13(8):695-701. 